\title{
LEFT-SIDED ORAL ASYMMETRIES IN SPONTANEOUS BUT NOT POSED SMILES*
}

\author{
Douglas R. Wylie† and Melvyn A. Goodale $\ddagger$ \\ Department of Psychology, University of Western Ontario, London, Ontario N6A 5C2, Canada
}

(Received 7 October 1987: accepted 9 February 1988)

\begin{abstract}
To investigate possible facial asymmetries during the production of posed and spontaneous smiles, the displacement of various reference points on the mouth were measured as subjects produced both kinds of smiles. Strobe cameras were used in combination with a computerbased analysis to record the smiles of left- and right-handed males and females. The analysis revealed that the left side of the mouth moved more than the right side during spontaneous but not posed smiles, supporting the notion that the right hemisphere may play a special role in emotional expression. This asymmetry was most apparent in left-handed females and right-handed males. These sex and handedness differences are discussed with reference to apparent inconsistencies in previous research on asymmetries in emotional expression.
\end{abstract}

\section{INTRODUCTION}

IN RECENT years, there have been several reports of facial asymmetry in the production of emotional expression. Several of these studies have found that the left side of the face was more expressive than the right, supporting the notion that the right hemisphere may play a special role in the control of emotional expression $[1-5,7,8,11,19,24,26,28,29]$. Most of these studies have involved rating composite photographs made up of the left side of the face and its mirror image or the right side of the face and its mirror image. On the basis of these ratings, the degree of asymmetry in facial expression has been estimated. With respect to smiling, the results of different studies using this technique have been largely contradictory with some suggesting that the left side of the face is more expressive or "intense" during smiling $[5,7,8,11,16,19]$ and others reporting no such asymmetries for smiling $\lfloor 21,28,29]$. Using direct observation, Chaurasia and Gaswami [9] reported that the left side of the face was more often the dominant side of a smile, at least in right-handers. They also found that left-handers showed an asymmetry in the opposite direction. This last finding is in direct contradiction to the work of CAMPBELL [8] who, using the composite photograph technique, found a greater incidence of a left-biased facial asymmetry in left-handers. Indeed, although both sex and handedness may be critical variables in this work, THOMPSON [32] has noted that previous studies have not included each of the four different sex and handedness groups or have failed to report the sex and handedness of those performing the expressions.

Another important variable determining the degree of asymmetry may be the

\footnotetext{
*This research was supported by grant No. MA 7269 from the Medical Research Council of Canada to M. A. Goodale.

$\dagger$ D. Wylie is now at Department of Psychology, Queen's University, Kingston, Ontario, Canada.

$¥$ To whom correspondence should be addressed.
} 
"spontaneity" of the smile. Using electromyography, ScHwarTz et al. [31] found that during the spontaneous expression of positive emotions, including smiling, there was greater activity in the right than in the left zygomatic major, one of the important muscles involved in smiling. No asymmetry was apparent during a posed condition however. On the basis of studies using his well-known Facial Action Coding System (FACS) [13], EKmaN et al. [14, 15] concluded that posed smiles show a left-sided bias while spontaneous smiles are much more symmetrical. These studies not only contradict one another but also the findings of DOPSON et al. [11], using the composite photograph technique, reported that both posed and spontaneous smiles are left-biased, with spontaneous smiles being the more asymmetric. To complicate things further, BoroD et al. [4] found no difference between posed and spontaneous expressions using composite photographs. Clearly, the technique used to measure the asymmetry of the smile, as well as the nature of the smile itself, appear to be critical variables in determining the amount and direction of asymmetry present.

The shortcomings of the composite photograph technique have been discussed in detail elsewhere $[25,32]$. One major criticism of this technique has centred on the possibility that there may be consistent differences in the external anatomy of the left and right sides of the face which bias the judgements of raters. A second criticism is that different raters might use very different criteria to judge whether a composite photograph is "happier" or whether a smile is more "intense".

HAGER and EKMAN [15] have criticized the use of EMG measurements which, they have argued, can be influenced by asymmetries in the tissue between the recording electrode and the muscle. At the same time, they have admitted that their Facial Action Coding System may itself not be a sensitive enough measure to detect low levels of asymmetry.

Recently, WOLF and GOODALE [34] developed a new movement analysis technique to measure very subtle degrees of oral asymmetry during speech and other oral movements. Using this method, they were able to demonstrate clear right-sided asymmetries in the production of both verbal and complex non-verbal movements of the mouth. Their technique involved making strobe-video recordings of the mouth during various oral movements and digitizing the mouth opening frame-by-frame using a computer-based analysis. The present study used this same technique to study oral asymmetry during the production of both spontaneous and posed smiles. Besides its sensitivity, this technique has an advantage over the techniques described above, in that a precise measure of the motor behaviour is made. As the mouth moves, measurements of the displacement of reference points on the oral apparatus are made with respect to the position of these same points when the face is at rest. Thus, this measurement takes into account the possibility that the face may be asymmetrical at rest $[18,22,27,30,35]$. Since asymmetries may manifest themselves before the movement is completed, measurements of the smile were made mid-way through the movement in addition to when the smile was most intense. It was hoped that the use of this very sensitive and direct measure of oral movement on different kinds of smiles made by subjects of different sex and handedness would help to sort out some of the confusion that exists in this area of research.

\section{METHOD}

Subjects

The subjects were 35 paid volunteers, 16 males ( 9 right-handed and 7 left-handed) and 19 females ( 9 right-handed and 10 left-handed). The mean age was $21.9 \mathrm{yr}$ with a range from 19 to $30 \mathrm{yr}$. Subjects were classified as righthanders if they wrote with their right hand and left-handers if they wrote with their left. The degree of handedness 
was determined by an 8-item questionnaire adapted from KIMURA [17]. If a right-handed subject did one or more of the activities listed (excluding writing) with the left hand, he or she was classified as a weak right-hander. If a lefthanded subject did two or more activities with the right hand (excluding writing), he or she was classified as a weak left-hander. All other subjects were classified as strong right- or left-handers. None of the subjects had any previous injury to the face or jaw, or wore dental braces. The subjects were participants in a study that measured oral asymmetries during the production of verbal and non-verbal movements as well as during smiling. This study reports only the results obtained from the data on smiling asymmetries.

\section{Procedure}

The procedure designed by WOLF and GoODALE [32] was used. Subjects were seated on a stool $60 \mathrm{~cm}$ high, facing a camera located approximately $2 \mathrm{~m}$ away. A Sony rotary-shutter camera equipped with a zoom lens (22.5-90 $\mathrm{mm})$ was used to provide clear images of the subject's mouth and nose at $60 \mathrm{~Hz}$. The subject's face was illuminated with a floodlight placed directly behind the camera. For protection, the subjects wore dark sunglasses. During testing, the subject was asked to fixate on a point just above the camera and to avoid movement of the head and body. The subject's mouth movements were recorded on a Sony SLO-325 video cassette recorder (Beta 1 speed). A TEL video counter-timer (10 msec revolution) was superimposed on each frame to provide a measure of elapsed time. The subject's image was observable on a TV monitor, visible only to the experimenter. A small microphone was attached to the subject's clothing to monitor the audio output.

Make-up was applied to enhance the contrast of the subject's mouth on the camera. A white-coloured make-up stick was applied to the lips and a black eyeliner pencil was used to outline the lips. The black eyeliner was also used to put reference points (i.e. crosses) on the subject's face. Crosses were placed on the centre of the tip of the nose and on both cheeks at a point $3 \mathrm{~cm}$ lateral to the tip of the nose. Similarly, crosses were put on the midline of the upper and lower lip, the corners of the mouth, and halfway between the corner and midline of either lip.

While spontaneous smiles occurred throughout the testing procedure, to ensure that enough samples of this behaviour were available, a second experimenter entered the room after about $10 \mathrm{~min}$ of testing and made a comment about the subject's rather unusual appearance. Invariably this elicited strong and apparently spontaneous smiling from the subject. Immediately following this, the subject was asked to pose facial expressions of six emotions including happiness, disgust, surprise, anger, sadness and fear (the order was counterbalanced across subjects). The subject was first told which emotion to express, while imagining a scenario (verbally cued by the experimenter) related to the given emotion. For happiness, the cue provided was "Somebody has given you a nice birthday gift". The subject also had the option to refer to a card containing a picture of an actor expressing the six emotions (from LaDavas et al. [20]). The subject performed each of the expressions three times. Thus, while the subject was asked to pose a number of emotional expressions, the target expression for this study was the posed smile.

\section{Videotape analysis}

The videotape records were analysed with a Sony Video Motion Analyser which allows stable single-frame advancement of the video image. Each frame of the videotape was projected onto a digitizing tablet interfaced with a microcomputer.

Only those smiles which occurred when the subject was not talking or looking away from the camera were analysed. An additional requirement was that the reference points be visible throughout the movement.

Each of the reference points on a sequence of frames comprising a movement was digitized on the tablet and stored in the computer as two-dimensional Cartesian coordinates. The reference points were digitized for three frames within each smile. These included the frame immediately preceding the onset of the smile (the first frame), the frame judged to correspond to the maximum intensity of the smile (the final frame), and the frame midway between these two (the middle frame). Measurements of the displacement of reference points on the mouth from the first frame to the middle frame, and from the first frame to the final frame were made with respect to the point on the nose which did not move during the smile. In this way, measurements were made of the displacement of the left and right corners of the mouth, and the points marking the middle of the left and right halves of the upper lip (hereafter referred to as the upper lip measure). The reliability of the measurements was checked by entering the data from the videotapes twice for 10 smiles chosen at random and calculating correlation coefficients on the different measures. The $r$-values were 0.89 and 0.88 for the corner and upper lip measurements respectively for the middle frame, and 0.81 and 0.82 from the for the final frame. To increase the reliability, each smile was analysed three times and the mean measure was used. Furthermore, when possible, two each of the spontaneous and posed smiles were analysed and their measures were averaged.

\section{RESULTS}

For some subjects, acceptable data were obtained from only one of the two categories of smiles. Therefore, to maximize the amount of available data, separate analyses were carried 
out on spontaneous and on posed smiles. In addition, a combined analysis was carried out on those subjects for whom data was available on both types of smiles.

Of the 9 right-handed females, 2 were classified as weakly right-handed. None of the 9 right-handed males were classified as weakly right-handed. Four of the 10 left-handed females and 4 of the 7 left-handed males were classified as weakly left-handed. Analysis of the data on asymmetries in smiling revealed no effects of this variable whatsoever. In short, whether or not an individual showed a strong hand preference across a varicty of tasks had no effect on mouth asymmetry during smiling. For this reason, this variable was not included in subsequent analyses.

\section{Spontaneous smiles}

At least one spontaneous smile was available for analysis in 32 of the subjects. Measurements of the upper lip movement and the corner movement were subjected to a $2 \times 2 \times 2 \times 2($ Sex $\times$ Handedness $\times$ Frame $\times$ Side $)$ repeated-measures analysis of variance with Sex and Handedness as between-group factors, and Frame (middle/final) and Side (left/right) as within-group factors. As can be seen in Table 1, the left corner of the mouth moved significantly more than the right corner $(F(1,28)=5.66, P<0.025)$. There was also a significant three-way interaction between Sex, Handedness, and Side $(F(1,28)=5.72$, $P<0.025)$. Examination of this interaction suggested that the left-handed females showed the greatest left-sided asymmetry. A left-sided asymmetry was also apparent in the righthanded males, while the asymmetry was much reduced in left-handed males and perhaps even reversed in right-handed females (see Table 1). Post-hoc comparisons between individual means (LSD at $P<0.05$ ) provided some support for these conclusions. There was also a three-way interaction between Handedness, Frame, and Side $(F(1,28)=7.44$, $P<0.011)$. A non-conservative post-hoc test (LSD) suggested that the left-biased asymmetry was present in the left-handers in the final frame but not in the middle frame, while the converse was true of the right-handers. As expected, there was also a main effect of Frame, demonstrating that there was a greater displacement from the first to the final frame, than from the first to the middle frame $(F(1,28)=69.93, P<0.0001)$.

Table 1. Mean displacement (mm) of the corners of the mouth and each side of the upper lip during spontaneous smiles

\begin{tabular}{|c|c|c|c|c|c|c|c|}
\hline \multirow[b]{2}{*}{ Measure } & \multirow[b]{2}{*}{ Frame } & \multirow[b]{2}{*}{ Side } & \multicolumn{2}{|c|}{ Males } & \multicolumn{2}{|c|}{ Females } & \multirow[b]{2}{*}{ Mcan } \\
\hline & & & Left-handed & Right-handed & Left-handed & Right-handed & \\
\hline \multirow{4}{*}{ Corner } & \multirow{2}{*}{ Middle } & Left & 5.3 & 5.2 & 4.6 & 3.3 & 4.5 \\
\hline & & Right & 5.4 & 4.2 & 4.2 & 3.0 & 4.1 \\
\hline & \multirow{2}{*}{ Last } & Left & 7.6 & 8.1 & 8.2 & 5.6 & 7.3 \\
\hline & & Right & 7.4 & 7.4 & 6.4 & 5.8 & 6.7 \\
\hline \multirow{4}{*}{ Upper lip } & \multirow{2}{*}{ Middle } & Left & 3.8 & 3.7 & 3.1 & 2.9 & 3.3 \\
\hline & & Right & 3.9 & 3.3 & 2.5 & 3.1 & 3.1 \\
\hline & \multirow{2}{*}{ Last } & Left & 5.6 & 6.3 & 4.9 & 4.7 & 5.3 \\
\hline & & Right & 5.5 & 6.1 & 4.1 & 4.9 & 5.1 \\
\hline$N$ & & & 6 & 8 & 9 & 9 & 32 \\
\hline
\end{tabular}


The measurement of the upper lip also revealed the main effect of Frame $(F(1,28)=80.48$, $P<0.0001$ ), but not Side. Nevertheless, the same three-way interaction of Sex, Handedness, and Side was revealed $(F(1,28)=4.16, P<0.05)$, with the same implications about the sex and handedness groups as with the corner measure (see Table 1).

\section{Posed smiles}

At least one acceptable posed smile was available for 31 of the subjects. As with the spontaneous smiles, a $2 \times 2 \times 2 \times 2$ repeated-measures ANOVA was perfomed on the measures of the upper lip movement and the corner movement. No main effects or interactions were revealed except the main effect of Frame (corner, $F(1,27)=118.29$, $P<0.0001$; upper lip, $F(1,27)=106.11, P<0.0001)$, and an interaction of Sex, Frame, and Side (upper lip measure) $(F(1,27)=4.37, P<0.044)$ suggesting that for the upper lip measure males showed a rightward asymmetry that was more prominent at the final than the middle frame, while females showed a leftward asymmetry that again was more prominent by the final than the middle frame (see Table 2).

Table 2. Mean displacement $(\mathrm{mm})$ of the corners of the mouth and each side of the upper lip during posed smiles

\begin{tabular}{|c|c|c|c|c|c|c|c|}
\hline \multirow[b]{2}{*}{ Measure } & \multirow[b]{2}{*}{ Frame } & \multirow[b]{2}{*}{ Side } & \multicolumn{2}{|c|}{ Males } & \multicolumn{2}{|c|}{ Females } & \multirow[b]{2}{*}{ Mean } \\
\hline & & & Left-handed & Right-handed & Left-handed & Right-handed & \\
\hline \multirow{4}{*}{ Corner } & \multirow{2}{*}{ Middle } & Left & 4.3 & 4.3 & 5.4 & 3.6 & 4.5 \\
\hline & & Right & 4.4 & 4.6 & 5.4 & 4.0 & 4.7 \\
\hline & \multirow{2}{*}{ Last } & Left & 6.4 & 7.1 & 8.5 & 6.5 & 7.3 \\
\hline & & Right & 6.4 & 7.7 & 8.2 & 6.7 & 7.4 \\
\hline \multirow{4}{*}{ Upper lip } & \multirow{2}{*}{ Middle } & Left & 2.8 & 3.6 & 4.2 & 3.7 & 3.7 \\
\hline & & Right & 3.0 & 3.1 & 4.0 & 3.6 & 3.5 \\
\hline & \multirow{2}{*}{ Last } & Left & 5.0 & 5.2 & 6.0 & 6.1 & 5.7 \\
\hline & & Right & 5.5 & 5.8 & 5.4 & 5.5 & 5.5 \\
\hline$N$ & & & 5 & 8 & 11 & 7 & 31 \\
\hline
\end{tabular}

\section{Posed vs spontaneous smiles}

At least one of each of the posed and spontaneous smiles were available for 27 of the subjects. To make a direct comparison of the two conditions, difference scores $[\mathrm{R}-\mathrm{L}]$ and ratio scores $[(R-L) /(R+L)]$ were calculated for each of the measures. These scores were then subjected to a $2 \times 2 \times 2$ (Sex $\times$ Handedness $\times$ Smile) repeated measures ANOVA with Sex and Handedness as between-group factors and Smile (spontaneous/posed) as a withingroup factor. As illustrated in Fig. 1, spontancous smilcs showed a left-biascd asymmetry while the posed smiles showed a right-biased asymmetry. This apparent difference between the direction of asymmetry in the two kinds of smiles was supported for the corner measure by a significant main effect of Smile at the middle frame for both the difference score $(F(1,23)=5.63, P<0.025)$ and the ratio score $(F(1,23)=4.70, P<0.040)$. This difference between spontaneous and posed smiles was also apparent at the final frame for the ratio score $(F(1,23)=4.48, \quad P<0.036)$, and approached significance for the difference score $(F(1,23)=3.35, P<0.077)$ (see Fig. 1). The main effect of Smile also reached significance for the upper lip measure at the final frame for the ratio score $(F(1,23)=4.90, P<0.035)$ and again approached significance for the difference score $(F(1,23)=3.70, P<0.064)$. Further- 
more, the main effect of Smile approached significance at the middle frame for the ratio score $(F(1,23)=3.42, P<0.075)$ (see Fig. 1). The difference scores for the upper lip measure taken from the middle frame for spontaneous smiles did not differ from those taken from the middle frame for posed smiles.

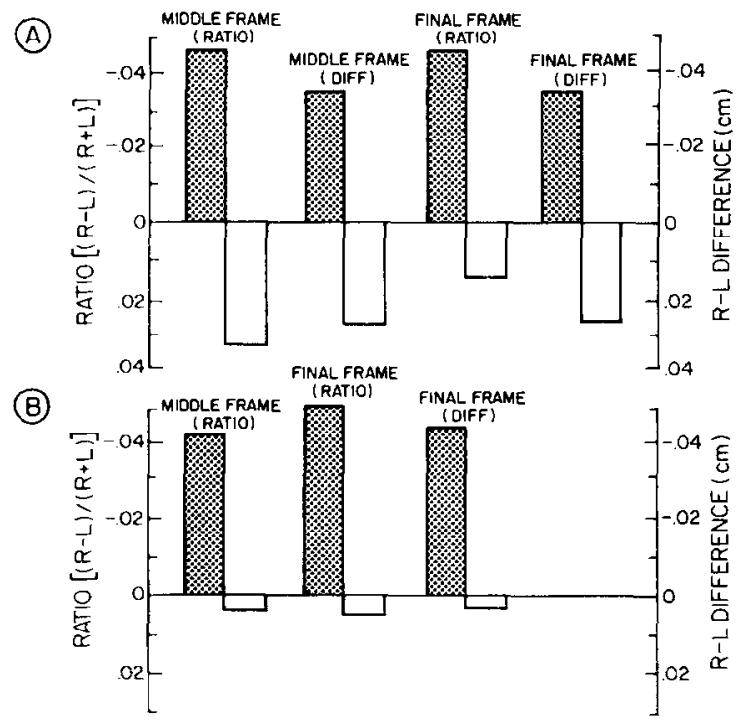

Fig. 1 Left-right ratio $[(R-L) /(R+L)]$ and difference scores $[R-L]$ for the movement of the corners of the mouth $(A)$ and the upper lip (B) during spontaneous and posed smiling for all subjects. Measures for both the spontaneous smiles (stippled bars) and the posed smiles (open bars) are illustrated for both the middle and the final frame. Notice that left-biased asymmetries are represented by bars above the zero line.

There were other significant effects and interactions for the corner measure. These included a main effect of Handedness at the final frame for the ratio scores $(F(1,23)=4.47$, $P<0.043$ ) suggesting that left-handers showed a left bias while right-handers show a smaller right bias. There was also a significant interaction between Sex and Handedness at the final frame for both the difference scores $(F(1,23)=6.85, P<0.015)$ and the ratio scores $(F(1,23)=4.48, P<0.043)$ suggesting that left-handed females showed a large left bias, right-handed females showed a large right bias, and both groups of males tended to show little bias. At the middle frame, there was a significant interaction between Handedness and Smile for the difference scores $(F(1,23)=6.39, P<0.018)$ and the ratio scores $(F(1,23)=6.86, P<0.015)$. Examination of the data suggested that spontaneous smiles in right-handers were left-biased, while their posed smiles were as equally right-biased. On the other hand, the posed and spontaneous smiles in left-handers were both slightly left-biased. Finally, there was also a three-way interaction of Sex, Hand, and Smile at the final frame for the difference scores $(F(1,23)=4.34, P<0.046)$. Examination of this interaction suggested that the spontaneous smiles were more left-biased compared to the posed smiles in all groups except the left-handed males (see Fig. 2). Moreover, this interaction once again demonstrates that the left-biased asymmetry in spontaneous smiles was primarily due to the biases present in the left-handed females and right-handed males. 


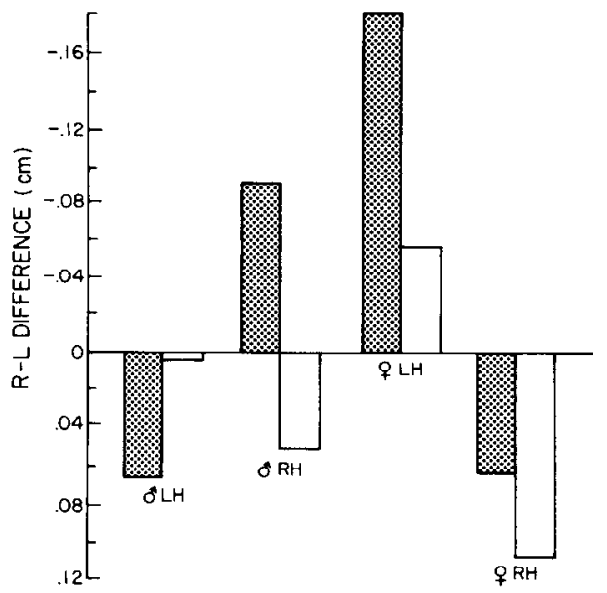

FIG. 2. Right-left difference scores [R - L] for the movements of the corners of the mouth taken from the final frame of the spontaneous and posed smiles produced by left- and right-handed males and females. The difference scores for the spontaneous smiles are illustrated by stippled bars; the differences scores for the posed smiles by open bars. Notice that left-biased asymmetries are represented by bars above the zero line.

\section{DISCUSSION}

The results of the present experiment show that during spontaneous, but not posed smiles, the left side of the mouth moves more than the right. Despite the fact that the asymmetries were often quite small, the use of a very sensitive measurement technique permitted the discrimination of subtle left-right differences. The present results are very similar to those of DOPSON et al. [11] who also found that spontaneous smiles were more left-biased than posed smiles but used a composite photograph technique rather than direct measurement. Moreover, DANE and THOMPSON [10] pointed out that in the experiment of DoPSON et al. [11] the ratings of the intensity of the left-left composites were significantly greater than the right-right composites for the spontaneous smiles but not for the posed smiles. Thus, there is even greater convergence between the results of the present study and those of Dopson $e t$ al. [11].

Movement asymmetries have typically been related to underlying left-right differences in brain organization. The right-sided oral asymmetries typically observed in speech, for example, have been interpreted as evidence of the special role the left hemisphere plays in the control of this complex behaviour [34]. There are, however, some additional problems in interpreting asymmetries in emotional expressions, which often involve much more than movements of the mouth. As noted by THOMPSON [32, 33] studies that have analyzed movements of the upper part of the face $[1,24]$, which receives a good deal of ipsilateral innervation, cannot as easily refer any observed asymmetries to cerebral specialization. Fortunately, since the measurements used in the present study involved only the oral apparatus, which is known to receive primarily contralateral neural innervation [25], the issue of hemisphericity can be addressed directly. The presence of a left-sided asymmetry in the production of spontaneous smiles supports the argument that the right hemisphere is specialized for the production of emotional expression, a notion which has found support in a number of other studies of the production of emotional facial expression $[1-3,5,7,8,11,24$, $28,29]$. Nevertheless, some of these studies have found that although a left-biased asymmetry 
exists for negative emotions, it is not always found for expressions of happiness [28, 29]. It must be remembered, however, that while the neural innervation of the mouth is largely crossed, there are some ipsilateral projections. Indeed, these ipsilateral projections may play a greater role in smiling than in other emotional expressions. One of the important muscles involved in smiling, the zygomatic major, receives some input from the ipsilateral hemisphere via the zygomatic branch of the temporofacial division of the facial nerve [25]. Thus, this ipsilateral input to one of the major effectors involved in smiling could depress any asymmetry that might be the result of greater activity in the crossed projections from the right hemisphere. Despite this, the left-sided asymmetry was still readily apparent in the spontaneous smiles of the present study, possibly because our measurement technique was highly sensitive.

Other interpretations of the left-sided asymmetry in the production of emotional expressions have been put forward. RINN [25], for example, has suggested that facial asymmetry may not reflect a specialization of the right hemisphere for emotion, but rather a function of the left hemisphere to inhibit emotion, which results in greater expressiveness in the left side of the face as the right side of the face is inhibited. RINN [25] has also suggested that positive emotions may not necessarily be inhibited, accounting for the fact that asymmetries are more apparent in negative emotions. While this explanation can account for much of the facial asymmetry data, it does not deal with those studies that have demonstrated perceptual asymmetries in the recognition of emotion (for review, see BRYDEN [6]). The argument that the right hemisphere has a special role to play in emotion is consistent with both the facial asymmetry data and the work on the perception of emotion.

The finding of an apparent asymmetry in the spontaneous but not in the posed smiles stands in direct opposition to the claim by Ekman and his colleagues that deliberate facial actions are left-biased while spontaneous facial expressions are symmetrical $[14,15]$. The resolution of this apparent conflict may lie in the kind of subjects used in their experiments. Close examination of our data suggested that the patterns of asymmetries were different in males and females and in left- and right-handers. The greatest left-biased asymmetries in spontaneous smiles were present in the left-handed females and the right-handed males. Right-handed females showed very little asymmetry in their spontaneous smiles. The presence of these individual differences in the direction and degree of asymmetry may help to explain the apparent conflict between our results and those of Ekman and his colleagues. Since HAGER and EKMAN [15] tested only right-handed females, it is not surprising that they found no asymmetry in their spontaneous smiles. An additional study by EKMAN et al. [14] (study 2) used females but did not report their handedness (although it is likely that they too were largely right-handed). Studies by SACKEIM et al. [29] and SACKEIM and GUR [28] also found no asymmetry in spontaneous expressions of happiness, but their study, which involycd rating composite photographs, included only photographs of right-handers, half of whom were female. (Although the authors report that the photographs were of posed smiles, EKMAN [12], who provided the photographs, contends that the smiles were spontaneous.) Thus, in these studies (as well as those by HAGER and EKMAN [15]), the group that showed the greatest asymmetry in spontaneous smiles in the present study (left-handed females) was not included.

The suggestion in the present study that male right-handers showed larger left-sided asymmetries than right-handed females is consistent with the findings of BoroD et al. [4], who in a study of right-handers, found positive expressions to be left-sided in males but not females. Furthermore, ChaURASIA and GASwami [9], who reported that the left side of the 
face is dominant during a spontaneous smile in right-handers, used a subject sample over $80 \%$ of which were male. CHAurasia and Gaswami [9] also found that the smiles produced by left-handers were less often left-biased. Again, however, over $87 \%$ of their left-handers were male, and in the present study left-handed males did not show a strong asymmetry.

At present, it is not clear what implications these different levels and directions of asymmetries in left- and right-handed males and females have for underlying differences in brain organization. One possibility, of course, is that there is greater right-sided cerebral asymmetry for emotional expression in left-handed females and right-handed males (who show the largest left-sided oral asymmetry in spontaneous smiles). Alternatively, the different groups may have similar patterns of lateralization of function at the level of cerebral cortex but different patterns of peripheral innervation. To sort out this question, it would be useful to examine asymmetries in the production of other spontaneous emotional expressions in left- and right-handed males and females and also to look systematically at the effects of unilateral brain damage on emotional expression as a function of sex and handedness.

Some studies have found left-biased asymmetries in posed smiles $[7,8,11,19]$. In these studies, the set of posed smiles might well have been contaminated with some spontaneous smiles. When asked to smile, subjects often smile spontaneously. It is possible that the inclusion of these more spontaneous and thus left-sided asymmetrical smiles could account for the finding of left-sided asymmetry in "posed" smiles. Of course, the same problem could have existed in the present study and could have attentuated the difference between posed and spontaneous conditions. Indeed the set of spontaneous smiles in this study may have been contaminated by some posed smiles. Some may even argue as to whether the spontaneous smiles in this study were in fact spontaneous. Several authors $[14,15,25,32]$ have cited neurological evidence suggesting that posed facial expressions originate in the motor cortex, while spontaneous expressions originate in sub-cortical structures. Moreover, it has been claimed that face to face conversation results in "social" smiles (presumably posed) originating in the motor cortex (cited in RINN [25]). Despite these complications, we are confident that the spontaneous smiles elicited in the present study are likely to have been spontaneous. Patients with central facial hemiparesis (the inability to produce a voluntary movement on one side of the central face) produce a bilateral smile in response to a joke, presumedly via undamaged sub-cortical structures involved in the generation of spontaneous emotional expressions [23]. Since the spontaneous smiles in the present study were elicited using a humorous incident, it is likely that the smiles obtained were indeed spontaneous. Like many situations in which smiles occur, the humour may have been tempered in some individuals by some embarrassment. Nevertheless, the present study provides clear evidence that the smiles produced in this way were more asymmetrical and left-biased than posed smiles. It is worth emphasizing, however, that the demonstration of this difference was dependent both on the use of a sensitive measure and on the inclusion of left- and right-handed subjects of both sexes.

\section{REFERENCES}

1. Alford, R. and Alford, K. F. Sex differences in the facial expression of emotion. Neuropsychologia 19, 605-608, 1981.

2. Borod, J. C. and CARON, H. S. Facedness and emotion related to lateral dominance, sex and expression type. Neuropsychologia 18, 237-241, 1980.

3. Borod, J. C., CARon, H. S. and Koff, E. Asymmetry of facial expression related to handedness, footedness, and eyedness: a quantitative study. Cortex 17, 381-390, 1981.

4. Borod, J. C. and KofF, E. Hemiface mobility and facial expression asymmetry. Cortex 19, 327-332, 1983. 
5. Borod, J. C., KoFF, E. and White, B. Facial asymmetry in posed and spontaneous expressions of emotion. Brain Cognit. 2, 165-175, 1983.

6. BKyuen, M. P. Laterality: Functional Asymmetry in the Intact Brain. Academic Press Inc., New York, 1982.

7. CAMPBell, R. Asymmetries in interpreting and expressing a posed facial expression. Cortex 14, 327--342, 1978.

8. CAMPBelL, R. Left-handers' smiles: asymmetries in the projection of a posed expression. Cortex 15, 571-579, 1979.

9. Chaurasia, B. D. and Goswami, H. K. Functional asymmetry in the face. Acta anatomica 91, 154-160, 1975.

10. Dane, F. C. and Thompson, J. K. Asymmetrical facial expressions: a different interpretation. Cortex 21, 301-303, 1985.

11. Dopson, W. G., Beckwith, B. E., Tucker, D. M. and Bullard-Bates, P. C. Asymmetry of facial expression in spontaneous emotion. Cortex 20, 243-251, 1984.

12. Ekman, P. Asymmetry in facial expression. Science 209, 833-834, 1980.

13. Ekman, P. and Friesen, W. V. Facial Action Coding System. Palo Alto, CA: Consulting Psychologists Press, 1978.

14. Ekman, P., Hager, J. C. and Friesen, W. V. The symmetry of emotional and deliberate facial actions. Psychophysiology 18, 101-106, 1981.

15. HaGer, J. C. and Ekman, P. The asymmetry of facial actions is inconsistent with models of hemispheric specialization. Psychophysiology 22, 1985.

16. Heller, W. and Levy, J. Perception and expression of emotion in right-handers and left-handers. Neuropsychologia 19, 263-272, 1981.

17. Kimura, D. Manual activity during speaking-II. Left-handers. Neuropsychologia 11, 51-55, 1973.

18. Koff, E., Borod, J. C. and White, B. Asymmetries for hemiface size and mobility. Neuropsychologia 19, $825-830,1981$.

19. Ladavas, E. The development of facedness. Cortex 18, 535-545, 1982.

20. Ladavas, E., Umilta, C. and RicCi-BItTi, P. E. Evidence for sex differences in right-hemispheric dominance for emotions. Neuropsychologia 18, 361-366, 1980.

21. LynN, J. G. and LynN, D. R. Smile and hand dominance in relation to basic modes of adaptation. J. abnorm. Soc. Psychol. 38, 250-276, 1943.

22. MCCurdy, H. Experimental notes on the asymmetry of the human face. J. abnorm. Soc. Psychol. 44, 553-555, 1949.

23. Monrad-Krohn, G. H. On the dissociation of voluntary and emotional innervation in facial paresis of central origin. Brain 47, 22-35, 1924.

24. Moscovitch, M. and OLdS, J. Asymmetries in spontaneous facial expressions and their possible relation to hemispheric specialization. Neuropsychologia 20, 71-81, 1982.

25. Rinn, W. E. The neuropsychology of facial expression: a review of the neurological and psychological mechanisms for producing facial expressions. Psychol. Bull. 95, 52-77, 1984.

26. Rubin, D. A. and Rubin, R. T. Differences in asymmetry of facial expression between left- and right-handed children. Neuropsychologia 18, 373-377, 1980.

27. SACKeIM, H. A. Morphological asymmetries of the face: a review. Brain Cognit. 4, 296-312, 1985.

28. SACKeIM, H. A. and GUR, R. C. Lateral asymmetry in intensity of emotional expression. Neuropsychologia 16, $473-481,1978$.

29. SACKeim, H. A., Gur, R. C. and SAuCY, M. C. Emotions are expressed more intensely on the left side of the face. Science 202, 434-435, 1978.

30. Sackeim, H. A., Weiman, A. L. and Forman, B. D. Asymmetry of the face at rest: size, area and emotional expression. Cortex 20, 165-178, 1984.

31. Schwartz, G. E., Ahern, G. L. and Brown, S. Lateralized facial muscle response to positive and negative emotional stimuli. Psychophysiology 16, 561-571, 1979.

32. Thompson, J. K. Right brain, left brain; left face, right face: hemisphericity and the expression of facial emotion. Cortex 21, 281-299, 1985

33. Thompson, J. K. Neuroanatomy, hemisphericity and facial asymmetry. Neuropsychologia 20, 699-701, 1982.

34. Wolf, M. E. and Goodale, M. A. Oral asymmetries during verbal and non-verbal movements of the mouth. Neuropsychologia 25, 375-396, 1987.

35. WolfF, W. The experimental study of forms of expression. Character and Personality 2, 168-176, 1933. 